\title{
A STUDY OF PERIOPERATIVE PAIN MANAGEMENT IN VASCULAR PATIENTS PRESENTING FOR LOWER LIMB AMPUTATION.
}

\author{
Dr. Venkatesan Duraiswamy (ST7 Trainee), A. Kotzadimitriou (Vascular Fellow), Dr. A. Malik (Cons), Dr. A.Krol (Cons) \\ St. George's University Hospital, London, UK
}

\section{Background and Aims:}

St. George's University Hospital is a tertiary referral centre for complex vascular surgery. The main aim of the study is to improve the pain management of vascular patients presenting for lower limb amputation; thereby improving the patient's satisfaction and experience during their stay in hospital. Our aim is to place a sciatic nerve catheter for all the patients having a lower limb amputation and their pain score should be $\leq 2$ according to our trust pain scale during the first 72 hours.

\section{Methods:}

This is a retrospective study and it is registered with the Hospital Clinical Audit Department (unique registration number CADB002486). We requested the Information Department to capture all the cases of lower limb amputations in vascular patients from May 2017 to April 2018, amputations in trauma patients were excluded. All data was collected from patient notes held in Medical Records and Electronic documentation management system. Retrospective data was collected from the nerve block local anaesthetic infusion chart, anaesthetic chart, drug chart and surgical care pathway. Forty-four patients were included in this study. Patient's notes with missing documentation were excluded. Pain scores were analysed based on a numerical rating scale of 0-4 ( $0=$ no pain at rest/on movement, $1=$ mild pain on movement, $2=$ moderate pain on movement, $3=$ severe pain on movement and $4=$ continuous pain at rest/severe pain on movement).

\section{Results:}

Out of 44 patients, 3 patients had Through knee amputation(TKA), 20 patients had Below Knee Amputation (BKA) and 21 patients had Above Knee Amputation (AKA). In total, 41(93\%) patients had a sciatic nerve catheter inserted either by anaesthetists under ultrasound guidance in $63.4 \%$ of patients or placed by surgeons under direct vision in $36.6 \%$ of patients. Out of 21 AKA patients, $17(70.8 \%)$ had a combined Sciatic and femoral nerve block. Thirty -seven patients $(84.1 \%)$ received patient-controlled analgesia or strong oral opioids in the postoperative period. On analysing pain scores, we noticed on day 1,34(82.9\%) patients had a pain score of $\leq 2$ and on day $2 \& 3,36(87.8 \%$ ) patients had pain score $\leq 2$ (figure 1). Preoperatively, sciatic nerve catheter was inserted only in 4 (9.1\%) patients. Postoperatively, 27(61\%) patients were admitted either in Intensive care unit or in High dependency unit, indicating patients in this cohort are associated with multiple comorbidities and high morbidity.

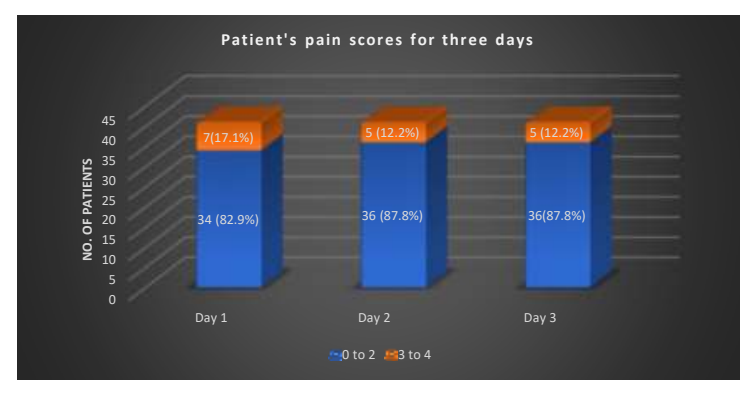

figure 1

\section{Conclusion:}

This study shows that sciatic nerve catheter LA infusion in lower limb amputation patients is associated with consistently low pain scores.. There are not many RCTs available regarding the use of sciatic nerve catheters in lower limb amputations and few trials have failed for many reasons e.g. unpredicted time of admission and lack of protocols. St. George's Hospital is a tertiary centre, performing more than 100 amputations a year. This has allowed us to collect sufficient evidence, resulting in the introduction of the Sciatic nerve block catheter pathway \& guidelines which have been put into clinical practice. This has led to an improved patient experience and quality of care. When compared to the previous audit in 2015-16, we significantly increased our Sciatic nerve catheter LA infusion service from $14 \%$ to $93 \%$. We are aiming to improve our service by inserting a sciatic nerve catheter preoperatively in these patients for better pain management. We are planning to standardise our postoperative analgesic regime in order to minimise the use of opioids.

\section{References:}

1. National Confidential Enquiry into Patient Outcome and Death, Lower Limb Amputation: Working Together, November 2014.

2. Malik A, Krol A Postoperative pain management in patients presenting for lower limb amputation. Audit of current practice in tertiary referral centre. ESRA7-0211, Reg Anesth Pain Med, Vol 42, N 5, suppl 1, Sep- Oct 2017

Funding and Competing interest:

None. 